\title{
Successful maintenance of process and outcomes for oesophageal cancer surgery in Ireland during the first wave of the COVID-19 pandemic
}

\author{
Jarlath C. Bolger ${ }^{1}$ (D) Noel E. Donlon ${ }^{1} \cdot$ Waqas Butt $^{1} \cdot$ Colm Neary $^{2} \cdot$ Mohammed Al Azzawi $^{3} \cdot$ Orla Brett $^{3}$. \\ Sinead King ${ }^{1}$ - Eithne Downey ${ }^{3}$ - Mayilone Arumugasamy ${ }^{3}$. Thomas Murphy ${ }^{2}$. William B. Robb ${ }^{3}$. Chris G. Collins ${ }^{4}$. \\ Paul A. Carroll ${ }^{4}$. Claire L. Donohoe ${ }^{1} \cdot$ Narayanasamy Ravi $^{1} \cdot$ John V. Reynolds ${ }^{1}$
}

Received: 5 February 2021 / Accepted: 9 March 2021 / Published online: 16 March 2021

(c) Royal Academy of Medicine in Ireland 2021

\begin{abstract}
Introduction The emergence of the novel coronavirus Severe Acute Respiratory Syndrome Coronavirus 2 (SARS-CoV-2) and the coronavirus disease COVID-19 has impacted enormously on non-COVID-19-related hospital care. Curtailment of intensive care unit (ICU) access threatens complex surgery, particularly impacting on outcomes for time-sensitive cancer surgery. Oesophageal cancer surgery is a good example. This study explored the impact of the pandemic on process and shortterm surgical outcomes, comparing the first wave of the pandemic from April to June in 2020 with the same period in 2019. Methods Data from all four Irish oesophageal cancer centres were reviewed. All patients undergoing resection for oesophageal malignancy from 1 April to 30 June inclusive in 2020 and 2019 were included. Patient, disease, and peri-operative outcomes (including COVID-19 infection) were compared.

Results In 2020, 45 patients underwent oesophagectomy, and 53 in the equivalent period in 2019. There were no differences in patient demographics, co-morbidities, or use of neoadjuvant therapy. The median time to surgery from neoadjuvant therapy was 8 weeks in both 2020 and 2019. There were no significant differences in operative interventions between the two time periods. There was no difference in operative morbidity in 2020 and 2019 ( $28 \%$ vs 40\%, $p=0.28$ ). There was no in-hospital mortality in either period. No patient contracted COVID-19 in the perioperative period.

Conclusions Continuing surgical resection for oesophageal cancer was feasible and safe during the COVID-19 pandemic in Ireland. The national response to this threat was therefore successful by these criteria in the curative management of oesophageal cancer.
\end{abstract}

Keywords COVID-19 · Oesophageal cancer · Oesophagectomy

\section{Introduction}

The emergence of the novel coronavirus Severe Acute Respiratory Syndrome Coronavirus 2 (SARS-CoV-2) and the coronavirus disease COVID-19 have had an enormous impact on nonCOVID-19 related hospital care. Disruption to surgical services

John V. Reynolds

reynoljv@tcd.ie

Trinity St. James's Cancer Institute, Dublin, Ireland

Mercy University Hospital, Cork, Ireland

Beaumont Hospital, Dublin, Ireland

University Hospital Galway, Galway, Ireland in Ireland and elsewhere due to seasonal viral illness is not a new phenomenon [1, 2]; however, the impact that COVID-19 has had on the provision of surgical services worldwide is unprecedented [3]. As the pandemic initially took hold, there were justified fears about increased perioperative mortality in patients who were suffering with COVID-19 or who were infected with SARSCoV-2 post-operatively [4-6]. There is an obvious requirement for strategies that allow risk stratification and the provision of safe elective surgical care. There are numerous advisory bodies publishing guidelines on how to provide surgical care during the COVID-19 pandemic [2]. Although these are sometimes conflicting, there is a clear consensus that separating services into COVID-19 and non-COVID-19 streams, or indeed separating hospital services into COVID-19 and non-COVID-19 hospitals, should be a key component of organisational strategy [2]. 
The impact of COVID-19 on a healthcare system depends on a number of factors, including the 'spike' in acute cases, the resilience of the healthcare system to deal with surges in patient numbers, and bed capacity (both ward-based and critical care) and safe staffing levels [2]. It has long been recognised that Ireland consistently exceeds the internationally recognised target of a maximum of $85 \%$ bed occupancy [7]. The addition of COVID-19 therefore brings a significant stressor to the Irish healthcare system and inevitably brings challenges in how we provide surgical services.

Surgery is the cornerstone for the curative treatment of oesophageal cancer, often combined with preoperative chemotherapy alone or combined with a combination of chemotherapy and radiation therapy for locally advanced disease. [8]. In contrast to squamous cell cancer of the oesophagus, where high dose radiation with chemotherapy, so-called definitive chemoradiation (dCRT), represents a valid alternative, this does not apply to adenocarcinomas which constitutes approximately $75 \%$ of the cancers managed in Ireland [8]. Furthermore, delaying treatment brings a significant risk of disease progressing from potentially curable to metastatic cancer.

Patients in the Irish population with oesophageal cancer tend to be older and have medical comorbidities, with a high incidence of cardiorespiratory disease, obesity, and diabetes [9]. When this is combined with complex major surgery with an inherent significant risk of perioperative cardio-pulmonary complications, and a risk of mortality of between 1 and 5\%, the added risk of mortality would be high if they acquired COVID-19 in the perioperative period.

As part of the response to the COVID-19 pandemic, the Irish government in conjunction with the Department of Health and the National Cancer Control Program (NCCP) temporarily leased capacity from the private hospital sector [10]. This allowed a significant, albeit temporary, increase in bed, intensive care, and operating theatre capacity, while services in the public sector were diverted to dealing with COVID-19. Initial reports have shown this strategy allowed surgical care to proceed in a safe manner on a broad front [10]. The aim of this study is to describe the impact of the pandemic's first wave on the delivery of surgery for oesophageal cancer. We report herein how these measures were successful in maintaining optimum pathways for patients, and achieving successful outcomes. This experience will also inform the delivery of such care in the current and potentially future waves of COVID-19 in Ireland.

\section{Methods}

A retrospective review of prospectively maintained databases in St James's Hospital (SJH) and Beaumont Hospital (BH) in Dublin, Mercy University Hospital (MUH) in Cork and University Hospital Galway (UHG), was performed.
Patients undergoing surgery with curative intent of oesophageal or oesophagogastric junction cancer between 1 April and 30 June 2020 were included and were compared with patients treated in the equivalent time period in 2019. In March 2020 SJH and BH were designated as COVID-19 treating hospitals. As part of the public-private partnership deal, the oeosophagogastric teams from $\mathrm{SJH}$ and $\mathrm{BH}$ had access to facilities in the Beacon Hospital Dublin, and the Blackrock Clinic in Dublin (designated as non-COVID-19 hospitals) to facilitate such operations. Operations proceeded in MUH and UHG without accessing the private system and separate pathways were introduced to facilitate time sensitive surgery separately to COVID-19-related care.

\section{Pre-operative approach}

All patients with a diagnosis of oesophagogastric cancer underwent: oeophagogastric-duodenoscopy with biopsy, endoscopic ultrasound (EUS) for local staging of oesophageal cancer, laparoscopy with peritoneal washings for junctional oesophageal cancers, and PET-CT, and were discussed at a multi-disciplinary meeting (MDM). In 2019, MDMs were conducted in person. From March 2020, delivery of MDMs switched to a secure video-conferencing platform to facilitate remote working and physical distancing. The pre-operative approach and work-up was not altered in spite of the COVID-19 pandemic.

\section{Treatment}

Some patients on 1 April were already on a treatment pathway using neo-adjuvant therapy. Where possible patients' treatment continued as planned. For those who had recently completed neoadjuvant treatment with curative intent, their pathway continued as planned. All urgent cancer care was separated from $\mathrm{SJH}$ and temporarily relocated to the Beacon Hospital. A proportion of urgent care was temporarily relocated from $\mathrm{BH}$ to the Blackrock Clinic, with some care continuing in $\mathrm{BH}$ on care pathways separate to COVID-19-related care. In the Beacon Hospital and Blackrock Clinic, inpatient care was facilitated by consultant and non-consultant staff from $\mathrm{SJH}$ and $\mathrm{BH}$ respectively for perioperative and post-operative care. Cancer surgery remained on site in MUH and UHG on new care pathways separate from COVID-19-related care. Outpatient consultations were provided remotely where deemed safe and appropriate by the relevant consultant. All patients physically attending an outpatient appointment underwent a pre-screening questionnaire and a temperature check. Patients and clinicians wore face coverings and maintained social distancing as much as appropriate during consultations. Patients who were planned 
admissions for surgery underwent naso-pharyngeal swabbing with polymerase chain reaction (PCR) testing at most $72 \mathrm{~h}$ prior to their admission. On admission they underwent a screening questionnaire designed to identify any new COVID-19 symptoms or any potential close contacts that would require a period of isolation. A lung CT scan was also performed within $48 \mathrm{~h}$ of surgery in selected cases (particularly in the early stages of the pandemic while the efficacy of PCR screening was being established), consistent with national guidelines, to ensure that no early signs of COVID were present in PCR negative patients. Where possible patients were treated in single bed bays both in the intensive care unit and on the ward for the duration of their stay.

\section{Analysis}

Analysis of data was performed using Graphpad Prism (v. 8.4.2, Graphpad Software, San Diego, USA). Categorical variables were compared using Fischer's exact test, and Mann-Whitney $U$ test was used for continuous variables.

\section{Ethics}

Approval from the local audit committee was obtained in each participating centre.

\section{Results}

During the first wave of the SARS-CoV-2 pandemic, 45 patients underwent surgery for oesophageal or oesophagogastric junction cancer, compared with 53 in the same time period in 2019. The groups were well matched (Table 1). There were no differences in age, gender distribution, BMI, or co-morbidity. There were no differences in the use of neoadjuvant therapy in either patient cohort, with most patients receiving either peri-operative chemotherapy or neoadjuvant chemoradiotherapy, as per the FLOT and CROSS protocols, respectively $[11,12]$. A particular concern was that the pandemic might delay access to time sensitive interventions, particularly surgery following perioperative therapy, but there was no difference in the time from finishing neoadjuvant therapy to definitive surgical intervention between the two groups ( 8 weeks in both 2019 and 2020; Table 1). In total, 28 patients had operations facilitated by the Beacon Hospital and Blackrock Clinic.

There were no significant differences in disease characteristics for those undergoing oesophagectomy in 2019 and 2020 (Table 2). Although concerns remain that patients may present later due to delaying seeking treatment, the majority of these patients were already undergoing investigation or on treatment pathways when the pandemic was beginning. The operative interventions undertaken were
Table 1 Demographics of patients undergoing surgery for oesophagogastric malignancy during the first wave of the COVID-19 pandemic and the corresponding time period in 2019

\begin{tabular}{llll}
\hline & $\begin{array}{l}\text { Oesophagectomy } 2019 \\
(n=53)\end{array}$ & $\begin{array}{l}\text { Oesophagectomy } 2020 \\
(n=45)\end{array}$ & $p$-value \\
\hline Mean age (range) & $63.8(38-86)$ & $64.5(46-82)$ & $0.72^{* *}$ \\
M:F & $37: 16$ & $37: 8$ & $0.2^{*}$ \\
ASA & & & \\
I & $5 / 53$ & $5 / 45$ & \\
II & $31 / 53$ & $30 / 45$ & \\
III & $17 / 53$ & $10 / 45$ & $0.55^{*}$ \\
IV & $0 / 53$ & $0 / 45$ & $0.06^{*}$ \\
Mean BMI & $27.9(19.9-37.2)$ & $26.5(19.2-36.5)$ & $0.33^{*}$ \\
Current smoker & $8 / 53(15 \%)$ & $3 / 45(7 \%)$ & $0.83^{*}$ \\
Ex-smoker & $20 / 53(38 \%)$ & $16 / 45(36 \%)$ & $1.0^{*}$ \\
Diabetes & $6 / 53(11 \%)$ & $5 / 45(11 \%)$ & $0.17^{*}$ \\
Hypertension & $26 / 53(49 \%)$ & $25 / 45(56 \%)$ & $0.52^{*}$ \\
Hyperlipidemia & $17 / 53(32 \%)$ & $17 / 45(38 \%)$ & $0.80^{*}$ \\
Neoadjuvant chemotherapy & $11 / 53(21 \%)$ & $11 / 45(24 \%)$ & $1.0^{*}$ \\
Neoadjuvant radiation therapy & $1 / 53(2 \%)$ & $0 / 45(0 \%)$ & $0.84^{*}$ \\
Neoadjuvant combination therapy & $25 / 53(47 \%)$ & $23 / 45(51 \%)$ & $0.65^{*}$ \\
No pre-operative treatment & $16 / 53(30 \%)$ & $11 / 45(24 \%)$ & $0.66^{* *}$ \\
Median time from NACTx/NACRTx to & 8 weeks $(4-23)$ & 8 weeks $(6-20)$ & \\
surgery & & & \\
\hline
\end{tabular}

*Fischer's exact test

**Mann-Whitney $U$ 
equivalent. The most common intervention in both 2019 and 2020 was 2 -stage en bloc oesophagectomy (38/53, 72\% and $34 / 45,76 \%$ respectively, $p=0.9)$. There was no difference in operative approach between 2019 and 2020 (open $30 / 53,57 \%$ and $23 / 45,51 \%, p=0.86$ ). Interestingly, in spite of early concerns about the safety of minimally invasive surgery and the potential for aerosolisation of virus, the number of minimally invasive operations was equivalent [13]. There was no difference in post-operative morbidity $(15 / 53,28 \%$ vs $18 / 45,40 \%, p=0.28)$, and there was no 90-day or in-hospital mortality in either time period. Post-operative length of stay was equivalent between the two time periods.

There were no instances of peri-operative COVID-19 infection, although one asymptomatic patient had PCR confirmed COVID-19 infection in the interval between finishing neoadjuvant therapy and progressing to surgery. This infection did not impact on their date of surgery.

\section{Discussion}

The Irish hospital system has never undergone a stressor such as the SARS-CoV-2 pandemic. For the delivery of surgery, there were credible fears that patients could not get surgery performed due to both the risk of vulnerable highrisk patients contracting SARS-CoV-2, with attendant high mortality [5, 6]. An initial report, of 1128 surgical patients, mostly for emergency surgery, with a perioperative diagnosis of COVID-19 (within seven days pre-operatively, or within 30 days post-operatively), reported a mortality rate of $25.6 \%$, with the highest risk in those who developed pulmonary
Table 2 Tumour characteristics, operative intervention, and 30-day outcomes for patients undergoing oesophagectomy between 1 April 2019 to 30 June 2019 and 1 April 2020 to 30 June 2020

\begin{tabular}{|c|c|c|c|}
\hline & $\begin{array}{l}\text { Oesophagectomy } 2019 \\
(n=53)\end{array}$ & $\begin{array}{l}\text { Oesophagectomy } 2020 \\
(n=45)\end{array}$ & $p$-value \\
\hline \multicolumn{4}{|l|}{ Tumour location } \\
\hline Upper/middle third & $4 / 53(8 \%)$ & $4 / 45(8 \%)$ & \\
\hline Lower third & $21 / 53(39 \%)$ & $18 / 45(35 \%)$ & \\
\hline Junctional (types I and II) & $24 / 53(45 \%)$ & $20 / 45(49 \%)$ & \\
\hline Other & $4 / 53(8 \%)$ & $3 / 45(8 \%)$ & $0.99 *$ \\
\hline \multicolumn{4}{|l|}{ Histology } \\
\hline Adenocarcinoma & $44 / 53(83 \%)$ & $38 / 45(86 \%)$ & \\
\hline SCC & $8 / 53(15 \%)$ & $6 / 45(11 \%)$ & \\
\hline HGD & $1 / 53(2 \%)$ & $1 / 45(3 \%)$ & $0.96 *$ \\
\hline \multicolumn{4}{|l|}{ Other } \\
\hline \multicolumn{4}{|l|}{ Operative intervention } \\
\hline Transhiatal oesophagectomy & $10 / 53(19 \%)$ & $6 / 45(13 \%)$ & \\
\hline 2-stage oesophagectomy & $38 / 53(72 \%)$ & $34 / 45(76 \%)$ & \\
\hline 3-stage oesophagectomy & $1 / 53(2 \%)$ & $1 / 45(2 \%)$ & \\
\hline Extended total gastrectomy & $4 / 53(8 \%)$ & $4 / 45(9 \%)$ & $0.90^{*}$ \\
\hline \multicolumn{4}{|l|}{ Operative approach } \\
\hline Open & $30 / 53(57 \%)$ & $23 / 45(51 \%)$ & \\
\hline Hybrid & $4 / 53(8 \%)$ & $4 / 45(9 \%)$ & \\
\hline Minimally invasive & $19 / 53(35 \%)$ & $18 / 45(40 \%)$ & $0.86^{*}$ \\
\hline 90-day morbidity & $15 / 53(28 \%)$ & $18 / 45(40 \%)$ & $0.28 *$ \\
\hline PPCs/Pneumonia & $14 / 53(26 \%)$ & $9 / 45(20 \%)$ & $0.42 *$ \\
\hline Anastomotic leak & $6 / 53(11 \%)$ & $4 / 45(9 \%)$ & $0.48 *$ \\
\hline ARDS & $3 / 53(6 \%)$ & $0 / 45(0 \%)$ & $0.24 *$ \\
\hline Other & $6 / 53(12 \%)$ & $5 / 45(11 \%)$ & $0.75^{*}$ \\
\hline Readmission to critical care & $3 / 53(6 \%)$ & $1 / 45(2 \%)$ & $0.62 *$ \\
\hline Re-intervention & $6 / 53(8 \%)$ & $6 / 45(13 \%)$ & $0.77 *$ \\
\hline In hospital mortality & $0 / 53$ & $0 / 45$ & $1.0^{*}$ \\
\hline 90-day mortality & $0 / 53$ & $0 / 45$ & \\
\hline Median length of stay & $13(7-56)$ & $12(6-55)$ & $0.38 *$ \\
\hline Peri-operative COVID-19 infection & N/A & $0 / 45$ & $\mathrm{n} / \mathrm{a}$ \\
\hline
\end{tabular}

n/a not applicable

*Fischer's exact test 
complications from COVID-19 [6]. In elective surgery with a perioperative diagnosis of COVID-19, the mortality rate was $18.9 \%$, again with a significant increase among those with pulmonary complications, particularly those that developed acute respiratory distress syndrome (ARDS) [6]. As a result of pulmonary complications $21.3 \%$ of patients were re-intubated. A significant although lower risk was reported in a Dutch propensity-matched study which demonstrated a perioperative mortality of $12 \%$ (compared with $4.6 \%$ in the non-COVID-19 population) in a mixed population of emergency and elective surgery [14].

Oesophageal cancer surgery carries significant risks, with major postoperative pulmonary complications (PPCs) the most significant cause of major morbidity and mortality [15]. Recent reports from Ireland show a 1\% overall in-hospital mortality rate, a $30 \%$ incidence of PPCs, $18 \%$ for pneumonia, 9.5\% for respiratory failure, and $2.7 \%$ for ARDS [15]. Clearly, the risks posed by SARS-CoV-2 in this population must be carefully mitigated to prevent increased perioperative mortality and life-threatening respiratory morbidity. An added risk of COVID-19 may be on increasing the risk of anastomotic leaks, as has been reported for colorectal cancer resections, as anastomotic leaks are reported in between 5 and $25 \%$ of oesophageal cancer cases and may have fatal consequences $[15,16,17]$. It has not yet been established if there is a causal link between COVID-19 infection and anastomotic leak, although given the incidence of COVID-related immune dysfunction and cytokine release, and microvascular compromise, the association appears plausible.

Notwithstanding, the data presented in this study showed no impact of COVID per se, or the national response to the pandemic, on key process metrics, as well as crucial perioperative outcomes such as PPCs, ARDS, respiratory failure, anastomotic leaks, and SARS-CoV-2 incidence postoperatively. The national response therefore appeared effective in this context.

The history of this response is of interest, as initially there was uncertainty among the surgical community nationally and internationally as to what constituted appropriate timely surgical care. A broad consensus did emerge however that where possible, elective operations should be postponed [2, 18, 19], and that cancer operations where possible should be delayed, neoadjuvant therapies expanded or extended, and definitive surgical intervention should be delayed at least if there was no non-surgical alternative [18]. These initial suggestions were based on a real fear of surgical safety but largely ignored the fact that little evidence supported alternative non-operative approaches, or extended neoadjuvant therapy, and prolonging time to surgery. As the narrative progressed, it was acknowledged that conservative nonoperative management of surgical illness should be guided primarily by evidence, patient and disease factors, rather than universally employed in an effort to avoid cases of
COVID-19 [20]. For oesophageal cancer, there is no data to suggest that surgery can be avoided for curatively treating adenocarcinoma of the oesophagus [8].

Safe pathways were therefore required. Based on the evidence, and common sense, having designated COVID19 'low-risk' hospitals where possible, universal patient perioperative PCR screening and judicious use of personal protective equipment can mitigate the impact of COVID-19 cases on elective operating lists $[10,18,19,21-23]$. The low-risk COVID-19 hospital or environment is supported as being effective in a large recent study of 500 patients where only $2 \%$ of patients developed COVID- 19 in the perioperative period [24]. In Ireland, through the Government response to the national emergency, and agreement from all key stakeholders involving contracts with private hospitals, patients requiring oesophageal cancer surgery in designated COVID-19 hospitals were in some cases relocated to private hospitals which were relatively low risk for COVID19 , and where access to ICU was not a barrier to delivery care in a timely manner. As we report herein, all patients had pre-operative PCR testing and selective CT thorax, and there was no postoperative COVID-19 infection during their inpatient stay. This reflects international experience [10, 24, 25 ] and suggests that continuing to provide urgent surgical oncology care is safe with a number of simple precautions. As services resume, it may be necessary to strengthen the public-private links to attenuate possible impaired access and surgical delays in the public system. This will require formal consultation, and political will to ensure surgery can proceed in a safe and timely fashion.

Although this study looks predominantly at disease factors, treatment pathways, and perioperative outcomes, it does not examine the psychological impact of the pandemic on patients undergoing cancer treatment. A recent study from India showed a significant impact on patients in a number of quality of life domains, with significant impacts on stress, anxiety, and depression [26]. Much of this impact was due to uncertainty surrounding treatment pathways as well as social isolation that comes with treatment during a pandemic. We must bear these lessons in mind as we attempt to structure safe care pathways for cancer patients during the current and any future pandemics.

This report highlights how the service for oesophageal cancer patients requiring complex cancer surgery was successfully maintained and safely delivered during the first wave. It is reassuring that our service offered a similar number of interventions in a matched period in 2019 as during the initial phase of the pandemic in 2020. Furthermore, there was no significant difference in timing of curative surgery following neoadjuvant therapy which is a surrogate marker for service efficiency. It is essential that we maintain these standards as the pandemic evolves. However, there remains significant risk of ongoing impact on surgical services [3, 
27]. Internationally, it has been estimated that over 2 million elective surgical procedures are being cancelled on a weekly basis as a result of COVID-19. There is potential for significant morbidity and early mortality as a result of delayed interventions and missed diagnoses [3].

A further concern, not evident at this point, but a probable legacy of this pandemic, is of a real concern about delayed diagnosis of malignancies [25]. It is important that the hospital system demonstrates the flexibility to adapt and reconfigure as patient needs change. Our study has demonstrated that highly complex surgery can safely be transferred into a centre with no prior experience of managing these patients with no impact on patient care. We demonstrated identical morbidity and mortality rates in the two time periods studied. This is reassuring and provides evidence for future reconfiguration or utilisation of the private hospital system should it prove necessary.

We acknowledge some limitations. It is a retrospective review of a time period with significant flux and uncertainty. Guidelines and attitudes of staff and patients evolved during the study period. It is not possible to account for whether these issues impacted on perioperative care and interventions in this cancer population. Although we have shown a similar level of activity pre- and during COVID19 , many of these patients were already on a curative treatment pathway including neoadjuvant therapy before the pandemic began. While these patients have progressed through the appropriate pathway, it is unclear how the patients with late or delayed diagnoses as a consequence of COVID-19 will fare. Others have also shown that some patients have changed their approach to treatments as a consequence of fear of contracting COVID-19 [25]. We have not accounted for that in this study, and it remains to be seen whether a similar attitude will emerge in our patient populace. In light of this, it is important that we continue to counsel patients adequately about the full spectrum of curative and palliative therapies for oesophagogastric malignancy so as patients can make informed decisions regarding their care.

As the SARS-CoV-2 pandemic evolves through the current second major wave of January 2021, it will continue to have a significant impact on elective surgery and potentially perioperative mortality $[3,27]$. Demonstrating the ability to maintain a safe, timely, and effective approach to major cancer surgery will be an important factor in managing hospital services through various peaks of the pandemic. Encouragingly, this study demonstrates how it can be done successfully, and this highly positive outcome should inform how management of the most complex of surgical cancer care can be managed safely and effectively through national planning and organisation even at a time of enormous stress to the health service nationally.

\section{Conclusions}

The SARS-CoV-2 pandemic poses major obstacles for maintaining essential cancer surgery. During the first wave of the pandemic, the Irish response was appropriate and supported continuing essential oesophageal cancer surgery. This offers a template for managing services during this or other future pandemics.

\section{Declarations}

Ethics approval Local audit approval was provided in each centre.

\section{References}

1. Cookson G, Jones S, Laliotis I (2017) Cancelled procedures in the English NHS: evidence from the 2010 tariff reform. Health Econ 26(12):e126-e139

2. Soreide K, Hallet J, Matthews JB et al (2020) Immediate and longterm impact of the COVID-19 pandemic on delivery of surgical services. Br J Surg 107(10):1250-1261

3. COVIDSurg Collaborative (2020) Elective surgery cancellations due to the COVID-19 pandemic: global predictive modelling to inform surgical recovery plans. Br J Surg 107(11):1440-1449

4. Aminian A, Kermansaravi M, Azizi S et al (2020) Bariatric surgical practice during the initial phase of COVID-19 outbreak. Obes Surg 30(9):3624-3627

5. Aminian A, Safari S, Razeghian-Jahromi A et al (2020) COVID19 outbreak and surgical practice: unexpected fatality in perioperative period. Ann Surg 272(1):e27-e29

6. COVIDSurg Collaborative (2020) Mortality and pulmonary complications in patients undergoing surgery with perioperative SARS-CoV-2 infection: an international cohort study. Lancet 396(10243):27-38

7. McDarby G, Smyth B (2019) Identifying priorities for primary care investment in Ireland through a population-based analysis of avoidable hospital admissions for ambulatory care sensitive conditions (ACSC). BMJ open 9(11):e028744

8. Kamarajah SK, Phillips AW, Hanna GB et al (2020) Definitive chemoradiotherapy compared to neoadjuvant chemoradiotherapy with esophagectomy for locoregional esophageal cancer: national population-based cohort study. Ann Surg May 19, Epub ahead of print

9. Donlon NE, Ravi N, King S, Cunninhgam M, Cuffe S, Lowery $M$ et al (2020) Modern oncological and operative outcomes in oesophageal cancer: the St. James's hospital experience, Ir J Med Sci 190(1):297-305

10. Bolger JC, Kelly ME, Whelan C et al (2020) Public-private partnership: strategies for continuing urgent elective operative care during the COVID-19 pandemic. Br J Surg 107(9):e320-e321

11. Al-Batran SE, Homann N, Pauligk C et al (2019) Perioperative chemotherapy with fluorouracil plus leucovorin, oxaliplatin, and docetaxel versus fluorouracil or capecitabine plus cisplatin and epirubicin for locally advanced, resectable gastric or gastrooesophageal junction adenocarcinoma (FLOT4): a randomised, phase 2/3 trial. Lancet 393(10184):1948-1957

12. Shapiro J, van Lanschot JJB, Hulshof M et al (2015) Neoadjuvant chemoradiotherapy plus surgery versus surgery alone for oesophageal or junctional cancer (CROSS): long-term results of a randomised controlled trial. Lancet Oncol 16(9):1090-1098 
13. Zheng MH, Boni L, Fingerhut A (2020) Minimally invasive surgery and the novel coronavirus outbreak: lessons learned in China and Italy. Ann Surg 272(1):e5-e6

14. Jonker PKC, van der Plas WY, Steinkamp PJ et al (2021) Perioperative SARS-CoV-2 infections increase mortality, pulmonary complications, and thromboembolic events: A Dutch, multicenter, matched-cohort clinical study. Surgery 169(2):264-274

15. Reynolds JV, Donlon N, Elliott JA et al (2021) Comparison of Esophagectomy outcomes between a National Center, a National Audit Collaborative, and an International database using the Esophageal Complications Consensus Group (ECCG) standardized definitions. Dis Esophagus 11;34(1):doaa060

16. Low DE, Kuppusamy MK, Alderson D et al (2019) Benchmarking complications associated with esophagectomy. Ann Surg 269(2):291-298

17. van der Werf LR, Busweiler LAD, van Sandick JW et al (2020) Reporting national outcomes after esophagectomy and gastrectomy according to the Esophageal Complications Consensus Group (ECCG). Ann Surg 271(6):1095-101

18. COVIDSurg Collaborative (2020) Global guidance for surgical care during the COVID-19 pandemic. Br J Surg 107(9):1097-1103

19. Elizabeth Brindle M, Gawande A (2020) Managing COVID-19 in surgical systems. Ann Surg 272(1):e1-e2

20. Kelly ME, Murphy E, Bolger JC, Cahill RA (2020) COVID-19 and the treatment of acute appendicitis in Ireland: a new era or short-term pivot? Colorectal Dis 22(6):648-649
21. Lancaster EM, Sosa JA, Sammann A et al (2020) Rapid response of an academic surgical department to the COVID-19 pandemic: implications for patients, surgeons, and the community. J Am Coll Surg 230(6): 1064-1073

22. Tuech JJ, Gangloff A, Di Fiore F et al (2021) Strategy for the practice of digestive and oncological surgery during the Covid-19 epidemic. J Visc Surg 157(3S1):S7-S12

23. Yeo D, Yeo C, Kaushal S, Tan G (2020) COVID-19 and the general surgical department-measures to reduce spread of SARSCOV-2 among surgeons. Ann Surg 272(1):e3-e4

24. Kasivisvanathan V, Lindsay J, Rakshani-Moghadam S et al (2020) A cohort study of 30 day mortality after NON-EMERGENCY surgery in a COVID-19 cold site. Int J Surg 84:57-65

25. Wahed S, Chmelo J, Navidi M et al (2020) Delivering esophagogastric cancer care during the COVID-19 pandemic in the United Kingdom: a surgical perspective. Dis Esophagus 33(9)

26. Rajan S, Akhtar N, Tripathi A et al (2021) Impact of COVID-19 pandemic on cancer surgery: patient's perspective. J Surg Oncol 123(5):1188-1198

27. Soreide K, Yaqub S, Hallet J et al (2021) A risk model of admitting patients with silent SARS-CoV-2 infection to surgery and development of severe postoperative outcomes and death: projections over 24 months for 5 geographical regions. Ann Surg 273(2):208-216 